\title{
Sputtering of polished EUROFER97 steel: surface structure modification and enrichment with tungsten and tantalum
}

\author{
Petter Ström ${ }^{a^{*}}$, Per Petersson ${ }^{a}$, Rodrigo Arredondo Parra ${ }^{b}$, Martin Oberkofler $^{b}$, \\ Thomas Schwarz-Selinger ${ }^{b}$, Daniel Primetzhofer \\ aDepartment of Fusion Plasma Physics, School of Electrical Engineering and Computer Science, \\ KTH Royal Institute of Technology, SE-10044 Stockholm, Sweden \\ bMax Planck Institute for Plasma Physics, \\ D-85748 Garching, Germany \\ cDepartment of Physics and Astronomy, Applied Nuclear Physics, \\ Uppsala University SE-75120 Uppsala, Sweden \\ ${ }^{*}$ Corresponding author \\ email: pestro@kth.se \\ Postal address: KTH, Fusion Plasma Physics, \\ Teknikringen 31, 114 28, Stockholm
}

\begin{abstract}
Surface structure modification and enrichment with tungsten and tantalum were measured for polished EUROFER97 samples after exposure to a deuterium ion beam. Time-of-flight medium energy ion scattering and time-of-flight elastic recoil detection analysis were implemented for measuring atomic composition profiles. Atomic force microscopy and optical microscopy were used to investigate surface morphology. The deuterium particle fluence was varied between $10^{21} \mathrm{D} / \mathrm{m}^{2}$ and $10^{24} \mathrm{D} / \mathrm{m}^{2}$, projectile energy was $200 \mathrm{eV} / \mathrm{D}$ and exposure temperatures up to $1050 \mathrm{~K}$ were applied. The average fraction of tungsten plus tantalum to total metal content in the $2 \mathrm{~nm}$ closest to the sample surface was increased from an initial 0.0046 to 0.12 for the sample exposed to the highest fluence at room temperature. The enrichment was accompanied by an increase in surface roughness of one order of magnitude and grain dependent erosion of the material. The appearance of protrusions with heights up to approximately $40 \mathrm{~nm}$ after ion beam exposure at room temperature was observed on individual grains. Samples exposed to $10^{23} \mathrm{D} / \mathrm{m}^{2}$ at temperatures of $900 \mathrm{~K}$ and $1050 \mathrm{~K}$ displayed recrystallization and cracking while changes to the total surface fraction of tungsten and tantalum were limited to less than a factor of two compared to the sample exposed to the same fluence at room temperature.
\end{abstract}

Keywords: EUROFER, sputtering, erosion, ToF-MEIS, AFM, ToF-ERDA 


\section{Introduction}

EUROFER97 was developed in the European Union as a reduced activation ferritic martensitic (RAFM) steel to be used in blanket modules for the ITER experiment $[1,2]$. Presently it is also considered for a future DEMO reactor [3,4]. Consequently, characteristics of EUROFER97 such as tensile strength, thermal creep properties, electrical, thermal and magnetic properties and behavior under neutron and proton irradiation have been investigated in previous works [5-8]. The nominal composition of EUROFER97 is 9.5 at.\% Cr, 0.5 at.\% C, 0.48 at.\% Mn, 0.33 at.\% W, 0.22 at.\% V and 0.043 at.\% Ta, balanced by Fe (atomic fractions converted from the weight fractions as reported in Ref. [7], different sources give slightly different numbers). It has been shown that both EUROFER and the similar steel F82H display reduced erosion rates after exposure to light ion bombardment $[9,10]$. This property can be understood from the presence of heavy elements with low sputtering yields, i.e. tungsten and tantalum, which are enriched at the surface by preferential sputtering [11] and thereby lower the overall sputter rate. Quantification of the thickness of the enriched layer as well as the fraction of tungsten in it has previously been attempted with model samples in the form of iron-tungsten films deposited on carbon or silicon. Methods employed to this end include Rutherford backscattering spectrometry (RBS) [12] and, to obtain a higher surface depth resolution, time-offlight medium energy ion scattering (ToF-MEIS) [13,14]. In the latter case, studies of the properties of the enriched layer at elevated temperatures have so far been complicated by diffusion of substrate material into the film [14]. Measurements of the enriched layer thickness and depth profiling of the tungsten and tantalum fraction in EUROFER97 after deuterium ion bombardment, with depth resolution of a few nanometers, have not been performed previously. The purpose of the present study is to provide such measurements and correlate their results to changes in surface morphology. The information can help improve the understanding of the material's response to plasma erosion. It can thereby help to guide design decisions on using RAFM steels in plasma exposed regions of future fusion machines, as suggested for example in Ref. [15]. We have employed ToF-MEIS and time-of-flight elastic recoil detection analysis (ToF-ERDA) to analyze the enriched layer of tungsten and tantalum produced in polished samples of EUROFER97 after exposure to a $600 \mathrm{eV}$ $\mathrm{D}_{3}{ }^{+}$ion beam. Deuterium particle fluences from $10^{21} \mathrm{D} / \mathrm{m}^{2}$ to $10^{24} \mathrm{D} / \mathrm{m}^{2}$ were applied at room temperature and for the fluence of $10^{23} \mathrm{D} / \mathrm{m}^{2}$, several samples were exposed 
at different temperatures up to a maximum of $1050 \mathrm{~K}$. Surface structure modification has been observed through optical microscopy and atomic force microscopy (AFM).

\section{Experimental}

\subsection{Sample preparation and exposure}

12 samples, $8 \times 10 \mathrm{~mm}^{2}$ and $1 \mathrm{~mm}$ thick were machined from a slab of EUROFER97 which was manufactured at Forschungszentrum Karlsruhe GmbH, Germany and delivered via the European Union's undertaking Fusion for Energy. Polishing was performed with 600 and 1200 grit sandpaper followed by diamond lapping films on a rotating disc. The grain sizes for the lapping films were successively $30 \mu \mathrm{m}, 15 \mu \mathrm{m}$, $9 \mu \mathrm{m}, 6 \mu \mathrm{m}$ and $3 \mu \mathrm{m}$. In the next step, an oil based $1.5 \mu \mathrm{m}$ diamond lapping paste was applied to a microfiber cloth which was used to polish the samples by hand. Finally, a $50 \mathrm{~nm}$ water based aluminum oxide suspension from Tedpella was used, also on a microfiber cloth. The samples were cleaned with water and ethanol after every polishing step. Following the final polishing step, washing was performed by first wiping the samples with a microfiber cloth soaked in ethanol and then submerging them in acetone for 20 minutes. Once removed from the acetone, the samples were wiped dry with non-abrasive optical lens tissue and baked at approximately $10^{-2}$ mbar and $130^{\circ} \mathrm{C}$ for 48 hours.

The exposure of the samples to a deuterium ion beam was performed with the recently assembled Second Ion Experiment for Sputtering and Thermal desorption Analysis (SIESTA) at IPP Garching, Germany. A discharge was set up in a deuterium plasma source which was placed at $3.6 \mathrm{kV}$ for samples numbered 1 and 2, and $4.7 \mathrm{kV}$ for the remaining samples. The increase of the source voltage was performed in order to increase the beam current and thereby reduce the exposure time for the samples. $\mathrm{D}_{3}{ }^{+}$ions were selected by means of a bending magnet and the resulting beam was impinging at normal incidence on the sample which was set at a potential $600 \mathrm{~V}$ lower than the source. Consequently the energy of the $\mathrm{D}_{3}{ }^{+}$ions when hitting the sample was $600 \mathrm{eV}$, or $200 \mathrm{eV} / \mathrm{D}$. The total ion fluence was measured via the time integrated current received by the sample and secondary electron emission was taken into account using biased Faraday shields surrounding the target platform. Beam currents between $9 \mu \mathrm{A}$ and $13 \mu \mathrm{A}$ onto the sample were reached, corresponding to a 
deuterium particle flux between $6.7 \times 10^{18} \mathrm{D} / \mathrm{m}^{2} \mathrm{~s}$ and $9.7 \times 10^{18} \mathrm{D} / \mathrm{m}^{2} \mathrm{~s}$ over a beam footprint area of $0.25 \mathrm{~cm}^{2}$. Heating via electron impact was applied using a tungsten filament placed behind the sample and temperature was measured both with a thermocouple pressed onto the back of the sample and with an infrared pyrometer monitoring the irradiated surface. The temperature was controlled within $\pm 70 \mathrm{~K}$. Table 1 gives the exposure conditions for the samples.

\begin{tabular}{|l|l|l|}
\hline Sample number & $\begin{array}{l}\text { Fluence } \\
{\left[\mathbf{D} / \mathbf{m}^{2}\right]}\end{array}$ & $\begin{array}{l}\text { Temperature } \\
{[\mathrm{K}]}\end{array}$ \\
\hline 1 & $10^{21}$ & 300 \\
\hline 2 & $10^{22}$ & 300 \\
\hline 3 & $10^{23}$ & 300 \\
\hline 4 & $10^{24}$ & 300 \\
\hline 5 & $5^{\times 10^{23}}$ & 300 \\
\hline 7 & 0 & 300 \\
\hline 9 & $10^{23}$ & 750 \\
\hline 10 & 0 & 300 \\
\hline 11 & $10^{23}$ & 900 \\
\hline 12 & $10^{23}$ & 1050 \\
\hline
\end{tabular}

Table 1: Exposure parameters for polished EUROFER97 samples at the SIESTA setup. Samples 1-5 constitute a fluence series at room temperature while samples 3, 9, 11 and 12 constitute a temperature series. Samples 6 and 8 were excluded from additional evaluation due to a misalignment of sample 6 in the setup causing the beam to partially miss the surface, and a carbon rich contamination layer on sample 8 that interfered with the intended sputtering experiment.

\subsection{Ion beam analysis}

After exposure, the samples were sealed in an argon atmosphere and shipped to Uppsala University where ToF-MEIS measurements were performed to quantify the surface enrichment with tungsten and tantalum, and obtain depth-profiles of the enriched layer. A description of the ToF-MEIS setup can be found in Ref. [16]. The primary beam of $60 \mathrm{keV} 4 \mathrm{He}^{+}$was impinging on the samples parallel to the surface normal and backscattered ions were detected at a scattering angle of $155^{\circ}$. The beam current was less than $10 \mathrm{pA}$ and the measurement time was shorter than $1000 \mathrm{~s}$. With 
a beam spot area of approximately $1 \mathrm{~mm}^{2}$, the corresponding maximum $4 \mathrm{He}^{+}$ion fluence is $6.2 \times 10^{16} / \mathrm{m}^{2}$, i.e. little enough not to have had any measurable effect on the composition of the samples. For some samples, several measurements were taken, varying the position of the ToF-MEIS beam spot within the SIESTA beam footprint. By this approach it was verified that the surface enrichment is homogeneous within the beam footprint for independent measurements averaged over the size of the ToFMEIS beam spot $\left(\sim 1 \mathrm{~mm}^{2}\right)$.

Measurements with ToF-ERDA were carried out primarily to quantify the amount of oxygen on the sample surfaces. A $36 \mathrm{MeV}$ beam of ${ }^{127 \mathrm{I}^{8+}}$ was employed at $67^{\circ}$ incidence with respect to the sample normal and recoils were detected at $45^{\circ}$ from the forward beam direction using the detection system described in Ref. [17].

\section{$\underline{2.3 \text { Microscopy }}$}

To investigate surface morphology changes resulting from the ion beam exposure, the samples were photographed through an optical microscope. The appearance of the surface was similar everywhere within the SIESTA beam footprint and images of $150 \times 150 \mu \mathrm{m}^{2}$ were recorded at points close to the center of the footprint.

AFM scans were performed on all samples both before and after ion beam exposure using a Dimension FastScan instrument from Bruker in tapping mode with a FastScan cantilever. The initial scans were done immediately after polishing, cleaning and baking. The scan size was $5 \times 5 \mu \mathrm{m}^{2}$ with $256 \times 256$ pixels and measurements were performed both at the center and at the edges of the samples. Two measurements used different scan sizes, also with $256 \times 256$ pixels, which showed similar surface features and average roughness as the rest of the measurements. The measurements performed after ion beam exposure used a scan size of $5 \times 5 \mu \mathrm{m}^{2}$ and $512 \times 512$ pixels.

\section{Analysis and results}

\subsection{Elemental composition and layer thickness from ToF-MEIS}

The raw data obtained with ToF-MEIS was energy converted and background subtracted by fitting K/E $\mathrm{E}^{1.5}$ to the background present at backscattered ion energies from $56.5 \mathrm{keV}$ to $6 \mathrm{o} \mathrm{keV}$ and subtracting the result from the entire spectrum (with 
free parameter $\mathrm{K}$, corresponding to a subtraction of white noise from the ToF spectrum). Fig. $1 \mathrm{a}$ and $\mathrm{b}$ show comparisons of the background subtracted spectra for the fluence series and the temperature series respectively. The spectra have been normalized so that their integrals from $19.5 \mathrm{keV}$ to $30 \mathrm{keV}$ are identical in order to facilitate comparison. The normalization does not affect the interpretation of the results as the overall signal height is not relevant for the analysis, but rather the relative heights of the parts of the spectrum corresponding to signals from different elements. For all cases where several measurements exist for the same sample, the displayed spectrum was constructed as the average of those measurements after background subtraction and normalization in order to improve measurement statistics and use all available data.

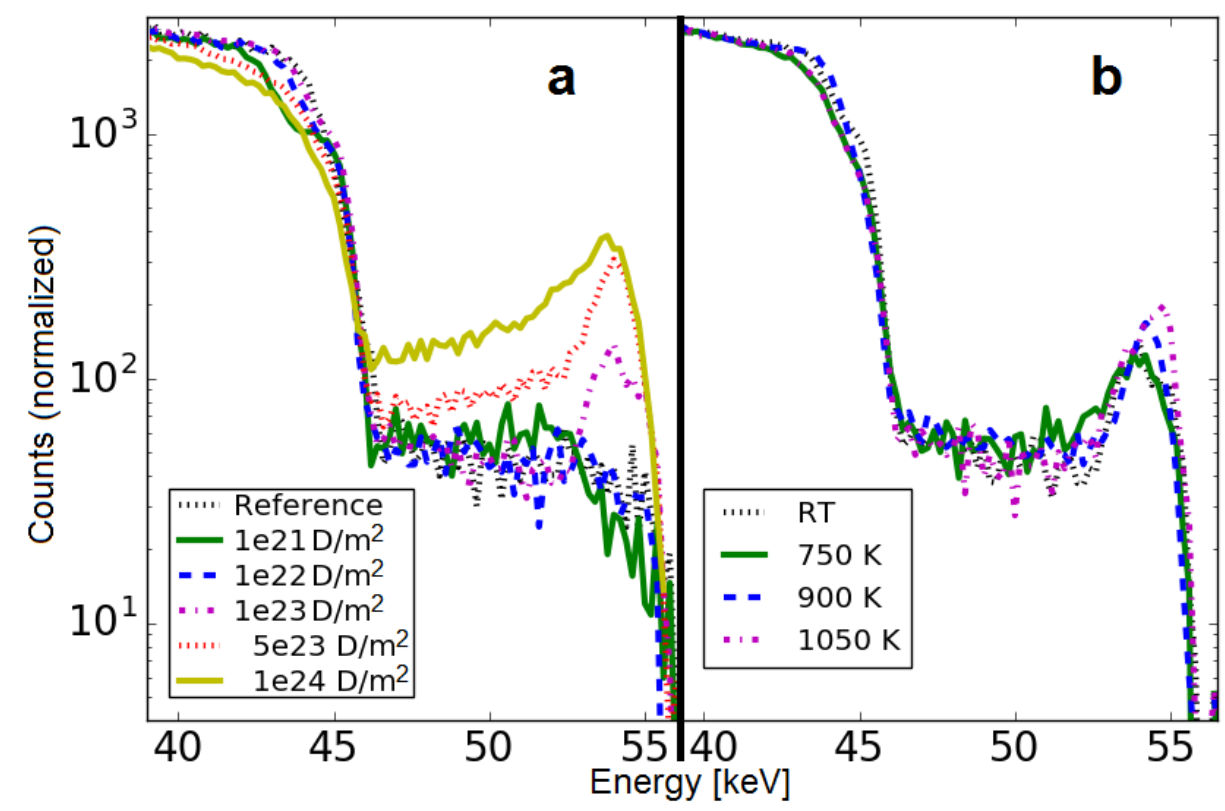

Figure 1: Energy converted and background subtracted ToF-MEIS spectra using a $60 \mathrm{keV} 4 \mathrm{He}^{+}$beam, from polished EUROFER97 samples exposed to a deuterium ion beam with (a) different fluences at room temperature and (b) a constant fluence of $10^{23} \mathrm{D} / \mathrm{m}^{2}$ at different temperatures.

A signal from tungsten and tantalum whose height depends on the atomic concentrations of those elements in the studied samples is seen in the present ToFMEIS spectra for energies below $55.2 \mathrm{keV}$; the energy of primary ions backscattered from tungsten at the sample surface. The tungsten and tantalum signals are indistinguishable and these elements are treated together for the remaining analysis. As such, numbers reported below consider the sum of tungsten and tantalum 
fractions. The spectra show peaking of the signal intensity close to $55.2 \mathrm{keV}$, which indicates an increased concentration of tungsten and tantalum close to the surface. Counts below $45.6 \mathrm{keV}$ come from primary ions backscattered from all elements in the sample, mainly iron and chromium, the principal constituents of the material. Analysis of the spectra from the fluence series was performed by means of comparison with simulations using the fast backscattering TRIM code TRBS [18]. Between $4 \times 10^{8}$ and $1.2 \times 10^{9}$ ions were simulated in each run and the energies of those ions backscattered in angles between $153^{\circ}$ and $157^{\circ}$ were recorded. A set of layers of $5 \mathrm{~nm}$ thickness each (or $\sim 4.1 \times 10^{16} \mathrm{at} / \mathrm{cm}^{2}$, see further discussion on depth scale conversion below), containing iron, tungsten and oxygen on top of an unmodified bulk were assumed for the initial simulations. The layer thicknesses as well as the concentrations of the three included elements were varied until the simulations yielded spectra fitting the experimental ones. It was assumed that all elements from vanadium to iron could be represented by iron for these simulations, that tungsten could represent both tungsten and tantalum, and that carbon is scarce enough in the samples to not contribute measurably to the ToF-MEIS spectra. The only one of these assumptions that gives a significant effect on the simulated spectra is replacing 9.5 at.\% of chromium with iron. Due to the difference in mass between these elements and the different stopping power in an iron-chromium mixture compared to pure iron, a $2.5 \%$ error in the signal height below $45.6 \mathrm{keV}$ for the simulations is produced. This is seen in the final result as a $2.5 \%$ error in the depth scale and the estimated atomic fractions of tungsten and tantalum.

In Fig. 2, the best fitting TRBS simulations are shown together with the measured spectra from the samples exposed to fluences between $10^{22}$ and $10^{24} \mathrm{D} / \mathrm{m}^{2}$. The fraction of tungsten averaged over the first $2 \mathrm{~nm}$ beneath the surface (actually representing the sum of tungsten and tantalum) to total metal content is indicated for each curve. A statement about the tungsten fraction in a thinner layer is not possible due to the surface depth resolution of ToF-MEIS in this case, which is approximately $2 \mathrm{~nm}$ [14]. While layers as thin as $2 \mathrm{~nm}$ are not included in the present simulations, significant variations of the tungsten concentration on that scale would be visible in the spectra. 


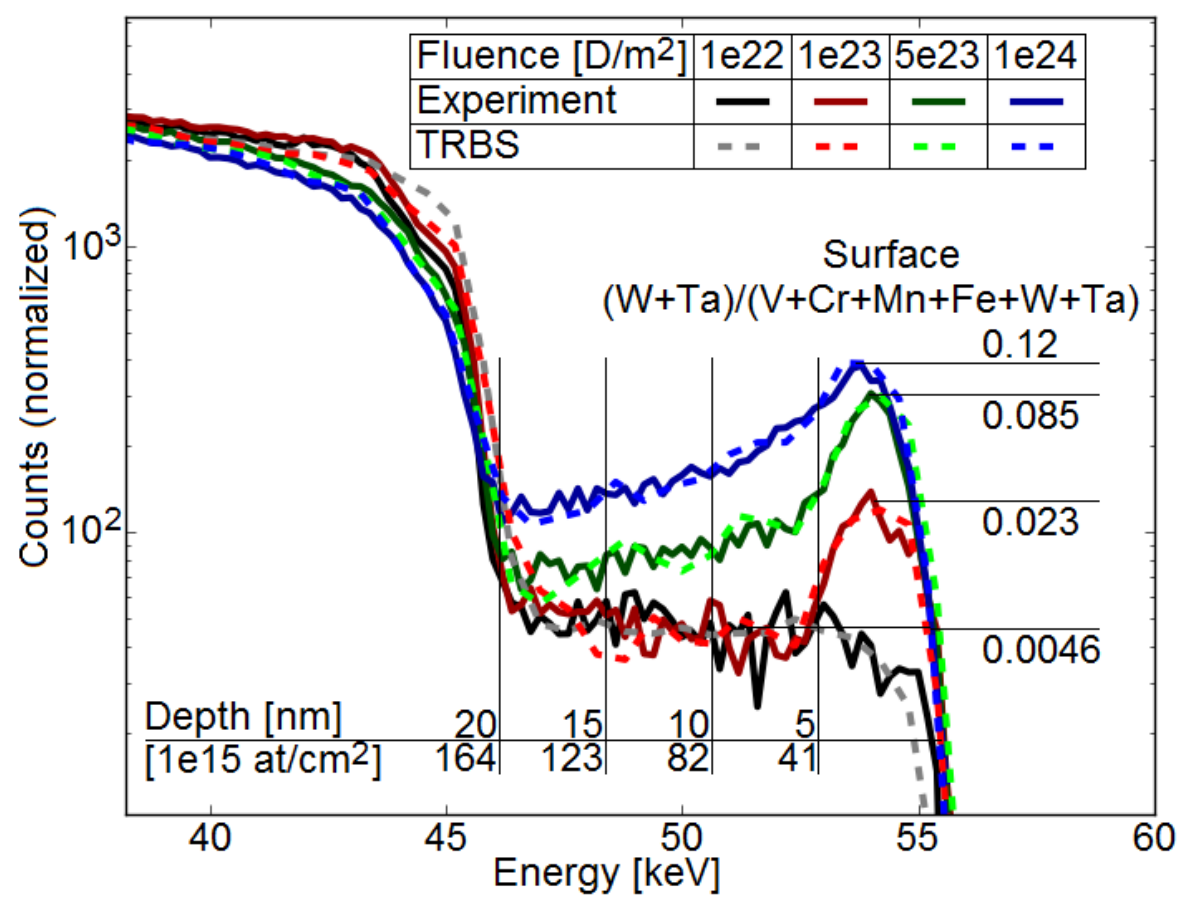

Figure 2: TRBS simulations compared to energy converted and background subtracted ToF-MEIS data from samples exposed to deuterium particle fluences between $10^{22} \mathrm{D} / \mathrm{m}^{2}$ and $10^{24} \mathrm{D} / \mathrm{m}^{2}$ at room temperature. The simulation results have been convolved with a Gaussian (FWHM $1.5 \mathrm{keV}$ ) to account for detector resolution, scaled to reproduce the iron signal from $30 \mathrm{keV}$ to $\sim 42 \mathrm{keV}$ and shifted by up to $0.2 \mathrm{keV}$ to coincide with the experimental spectra compensating for calibration offset. The depth scale given beneath the curves shows the approximate sample depth corresponding to a given backscattered particle energy. Conversion between $\mathrm{nm}$ and at $/ \mathrm{cm}^{2}$ is based on the density estimated for the sample exposed to $10^{24} \mathrm{D} / \mathrm{m}^{2}$, from which the curve with the highest tungsten and tantalum signal originates. Lower lying curves correspond to samples exposed to successively lower deuterium particle fluences.

The numbers for surface fractions given in Fig. 2 correspond to the sum of atomic fractions of tungsten and tantalum without taking surface oxide and carbon into account. Surface oxide was, however, taken into account in the simulations. As such, two free parameters are present when attempting to reproduce the measured spectra; the atomic fractions of tungsten and oxygen. To fit the measured signals from both tungsten and iron, both parameters are necessary. For the curves in Fig. 2, care has been taken to reproduce the tungsten signal but also to make sure that the simulation is in agreement with the iron signal at $45 \mathrm{keV}$ for samples exposed to $10^{23} \mathrm{D} / \mathrm{m}^{2}$ and 
above. The sample exposed to $10^{22} \mathrm{D} / \mathrm{m}^{2}$, for which no measurable surface enrichment is seen, was treated differently. Focus for this sample was rather on finding the bulk tungsten fraction. Therefore the surface oxide has not been carefully treated for the corresponding curve, and a discrepancy between measurement and simulation can be seen primarily at the iron signal edge. For the samples where surface enrichment is seen, the tungsten and tantalum concentrations drop toward the bulk values beneath the surface. A scale is overlain in Fig. 2 showing the approximate characteristic depth over which the concentration reduction takes place, both in $\mathrm{nm}$ and at $/ \mathrm{cm}^{2}$. The conversion of the depth scale between these units requires knowledge of the atomic density in the material, or the mass density and average molar mass. For the present TRBS simulations, the density of each layer was calculated individually by using an ideal bulk approximation and weighting the natural densities of pure tungsten and iron $\left(19.25 \mathrm{~g} / \mathrm{cm}^{2}\right.$ and $\left.7.87 \mathrm{~g} / \mathrm{cm}^{2}\right)$ by their respective atomic fractions. Oxygen was taken into account by multiplying the density of iron with the ratio of oxygen and iron average atomic masses, giving a value of $2.25 \mathrm{~g} / \mathrm{cm}^{2}$, which was then also weighted by the atomic fraction of oxygen in the layer. The depth scale conversion used for Fig. 2 is based on the layer densities from the sample exposed to $10^{24} \mathrm{D} / \mathrm{m}^{2}$ but the conversion is similar for all samples. For the sample exposed to $10^{23} \mathrm{D} / \mathrm{m}^{2}$, the simulated layer with tungsten and tantalum enrichment is $4.6 \mathrm{~nm}$ thick (c.f. for example Ref. [13] and [14]). The samples exposed to $5 \times 10^{23}$ and $10^{24} \mathrm{D} / \mathrm{m}^{2}$ seem to have been affected down to at least $20 \mathrm{~nm}$ of depth, however to interpret this statement the surface morphology changes induced by the exposure of the samples to the SIESTA ion beam must also be considered. This analysis is continued in sections 3.3 and 4 . We note that the atomic fraction of tungsten and tantalum in the unexposed sample, as well as those exposed to fluences low enough to not give any measurable surface enrichment, is measured to be 0.46 at.\% here rather than the 0.38 at.\% expected from the nominal composition. This number was verified by an additional measurement with Rutherford backscattering spectrometry (RBS) on an unexposed reference sample, which yielded 0.44 at.\%. The discrepancy between the nominal concentration and the measured one may be due to some irregularity in the material production or surface accumulation of tungsten and tantalum during machining and polishing of the samples. Discrepancies between the simulation result and the experimental data seen especially at backscattered ion energies between $47 \mathrm{keV}$ and $53 \mathrm{keV}$ for the curve corresponding to $5 \times 10^{23} \mathrm{D} / \mathrm{m}^{2}$ are 
due to the fact that the simulation is based on a set of discrete layers, while the measured curve corresponds to a smooth change of atomic concentrations.

\subsection{Surface oxide from ToF-ERDA}

The result obtained with ToF-ERDA on the sample exposed to $10^{24} \mathrm{D} / \mathrm{m}^{2}$ is displayed in Fig. 3. Detected elements are indicated and similar spectra were recorded on all samples included in the present study. In addition to the components of EUROFER97 and the expected surface oxide, some surface counts from hydrogen were noted.

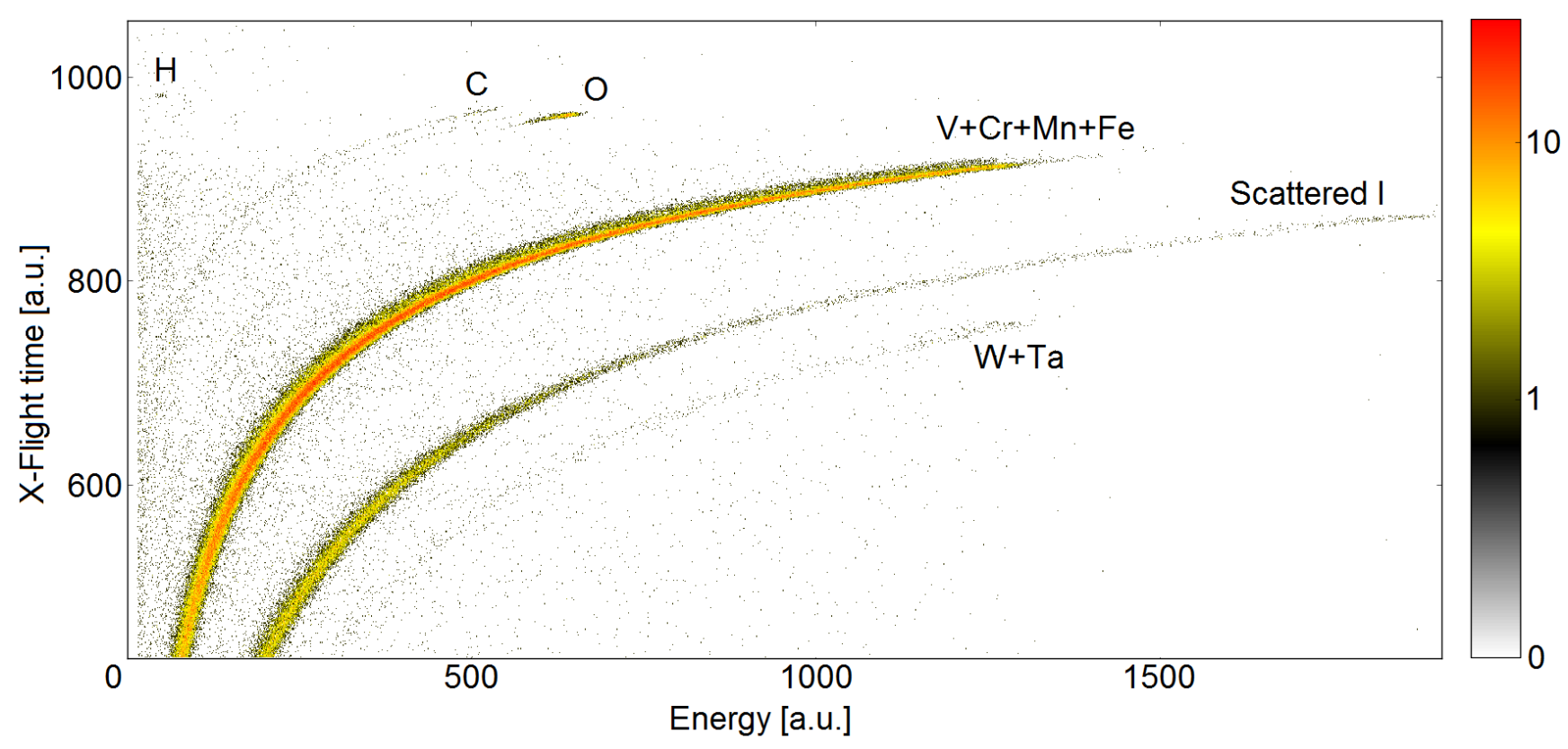

Figure 3: ToF-ERDA spectrum using a $36 \mathrm{MeV}^{127 \mathrm{I}^{8+}}$ beam, from the sample exposed to $10^{24} \mathrm{D} / \mathrm{m}^{2}$ at room temperature. The presence of surface oxide is indicated.

Depth profile conversions of the raw data were performed using Potku [19]. An oxygen peak was observed within the first $2.3 \times 10^{17} \mathrm{at} / \mathrm{cm}^{2}$ below the surface in the thus produced depth profiles, and the areal density of oxygen was found by integrating over that peak. This procedure yielded values between $1.5 \times 10^{16} \mathrm{at} / \mathrm{cm}^{2}$ and $5.8 \times 10^{16} \mathrm{at} / \mathrm{cm}^{2}$. The highest value of $5.8 \times 10^{16} \mathrm{at} / \mathrm{cm}^{2}$ was measured on the sample exposed to $10^{24} \mathrm{D} / \mathrm{m}^{2}$ which was also showing the largest surface roughness after exposure (see Fig. 5 d), whereas the lowest values of $1.5 \times 10^{16} \mathrm{at} / \mathrm{cm}^{2}$ and $1.7 \times 10^{16}$ at $/ \mathrm{cm}^{2}$ were found for samples 11 and 9, both of which were exposed at elevated temperature. All other samples showed intermediate concentrations of oxygen, with 
no clear correlation to their respective exposure conditions. For the TRBS simulated spectra shown in Fig. 2 the total areal densities of oxygen are $1.7 \times 10^{16}, 4.5 \times 10^{16}$ and $5.1 \times 10^{16}$ at $/ \mathrm{cm}^{2}$ respectively for the samples exposed to $10^{23}, 5^{\times 10^{23}}$ and $10^{24} \mathrm{D} / \mathrm{m}^{2}$. With both analysis methods discussed here, the amount of surface oxide seems to be highest on the samples exposed to the highest deuterium particle fluences. The oxide most likely originates from handling of the samples in air after the exposure at SIESTA and a possible explanation for the correlation is that the samples with higher surface roughness after exposure bind more oxygen per projected unit area. A further discussion on surface oxide and its implications for the present measurements follows in section 4. The fact that the oxygen concentrations from the best-fitting ToF-MEIS simulations are similar to what is found with ToF-ERDA strengthens the validity of the analysis.

\subsection{Evolution of surface morphology}

Changes to the surface were detected with optical microscopy on the samples exposed to $5 \times 10^{23}$ and $10^{24} \mathrm{D} / \mathrm{m}^{2}$ at room temperature and the sample exposed to $10^{23} \mathrm{D} / \mathrm{m}^{2}$ at $900 \mathrm{~K}$. Representative images from these samples, one unexposed reference sample and the one exposed to $10^{23} \mathrm{D} / \mathrm{m}^{2}$ at room temperature are given in Fig. 4 .

The data obtained with AFM was processed using the open source software Gwyddion to perform a mean plane subtraction and a row alignment by fitting and subtracting a $1^{\text {st }}$ degree polynomial from each horizontal line of pixel values, after which surface roughness was quantified. Two representative images from the initial set of scans, from samples numbered 1 and 9, are shown in Fig. 5 a and b. The surface structure seen in these images is dominated by polishing lines with depths from a few nanometers up to a maximum on the order of $10 \mathrm{~nm}$. The RMS deviation from the mean plane, $\mathrm{S}_{\mathrm{q}}$, was calculated for each scan. Before ion beam exposure all measurement points on the samples included in the present study showed values of $\mathrm{S}_{\mathrm{q}}$ between $0.99 \mathrm{~nm}$ and $2.65 \mathrm{~nm}$. The average value was $1.64 \mathrm{~nm}$. After exposure, the measurements on the samples exposed to $10^{21}$ and $10^{22} \mathrm{D} / \mathrm{m}^{2}$ showed no signs of surface modification. Above $10^{23} \mathrm{D} / \mathrm{m}^{2}$, however, significant morphology changes were detected in the samples exposed at room temperature. These are exemplified in Fig. $5 \mathrm{c}$ and $\mathrm{d}$ for $1 \mathrm{O}^{23}$ and $1 \mathrm{O}^{24} \mathrm{D} / \mathrm{m}^{2}$, which can be compared to the unexposed samples in Fig. 5 a and b. At $10^{23} \mathrm{D} / \mathrm{m}^{2}$, discrete boundaries between grains eroded to 
different heights become visible, but the overall surface roughness is within the range given above for the unexposed samples. At $10^{24} \mathrm{D} / \mathrm{m}^{2}$ surface roughness has increased by one order of magnitude. Protrusions with average height around $15 \mathrm{~nm}$ and a maximum height of approximately $40 \mathrm{~nm}$, similar to those observed in previous works $[14,20,21]$ are seen. Changes to the surface structure were also noted for the samples exposed to the highest temperatures of $900 \mathrm{~K}$ and $105 \mathrm{oK}$, shown in Fig. 5 e and $f$.

\section{Discussion}

The surface structure seen in Fig. $4 \mathrm{c}$ and d, which is better resolved in Fig. 5 d, should be taken into account in the interpretation of the ToF-MEIS results from the samples exposed to $5 \times 10^{23}$ and $10^{24} \mathrm{D} / \mathrm{m}^{2}$. The increase of surface roughness on these samples can help explain the apparent depth profiles seen in the ToF-MEIS spectra. Two specific features are noted. First, different grains are eroded at different rates. This can be observed in Fig. 4 a to $d$ as a more pronounced appearance of a grain structure for higher fluences, and in Fig $5 \mathrm{c}$ as a height difference between the surfaces of neighboring grains. Second, protrusions appear after exposure to $10^{24} \mathrm{D} / \mathrm{m}^{2}$ at room temperature on some grains, whereas other grains appear smooth as seen in Fig. 5 d. The sizes of these grains are on the order of a few $\mu \mathrm{m}$ up to approximately $10 \mu \mathrm{m}$ (see Fig. $4 \mathrm{c}-\mathrm{d}$ and $5 \mathrm{c}$-d). While the height variations between individual grains do not modify the ToF-MEIS spectra significantly thanks to the relatively large size of the grains compared to the height variation, the surface structure on individual grains does. Due to the increased surface roughness and protrusions, it becomes less meaningful to talk about a depth profile of the enriched layer modelled as a change in concentration beneath a flat surface for the samples exposed to the highest fluences. 
a) Unexposed reference

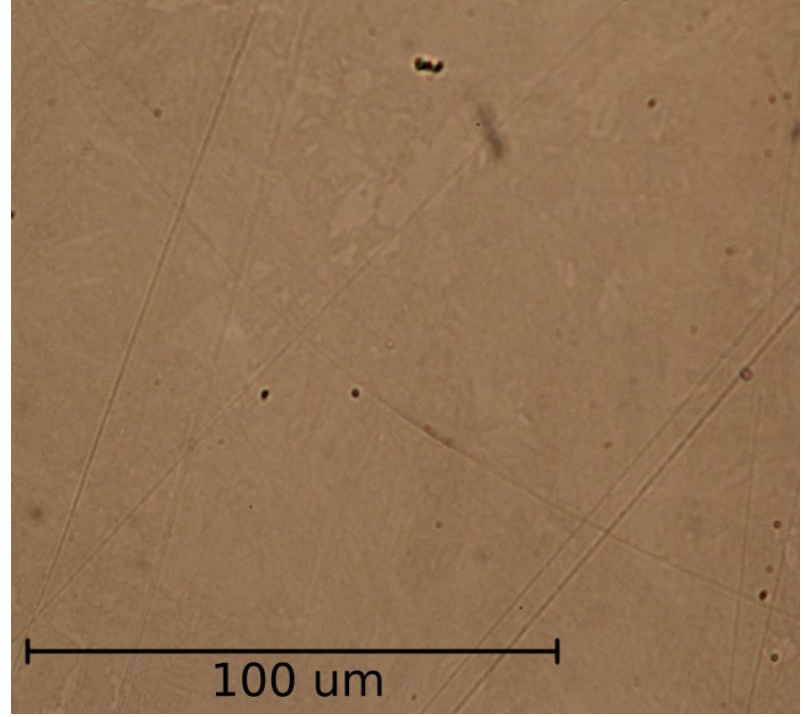

C) $5 \dot{e} 23 \mathrm{D} / \mathrm{m}^{2}, \mathrm{R} \cdot \mathrm{T}$

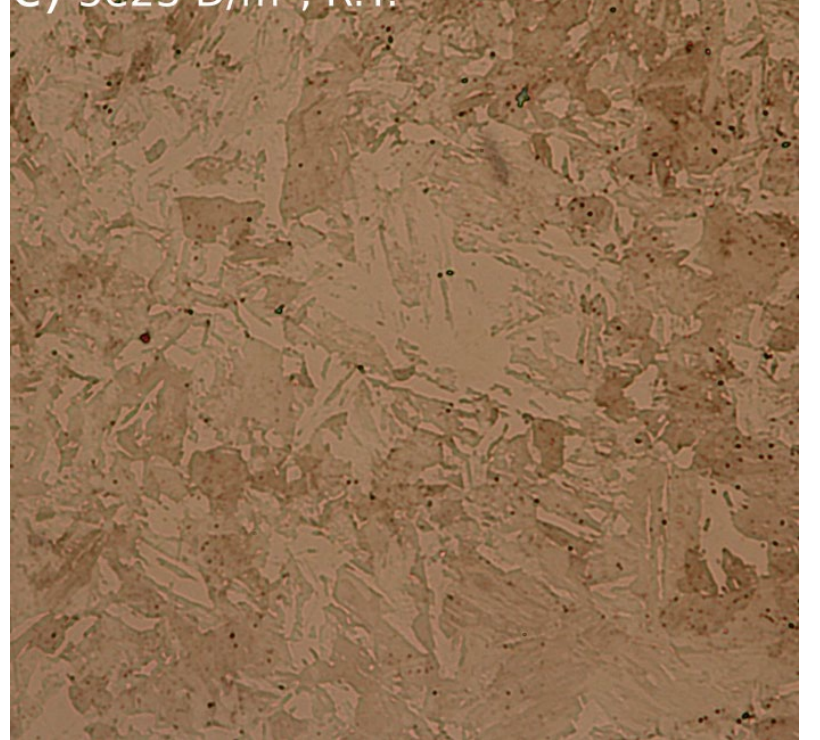

e) $1 \mathrm{e} 23 \mathrm{D} / \mathrm{m}^{2}, 900 \mathrm{~K}$ b) 1 e23 D/m², R.T.
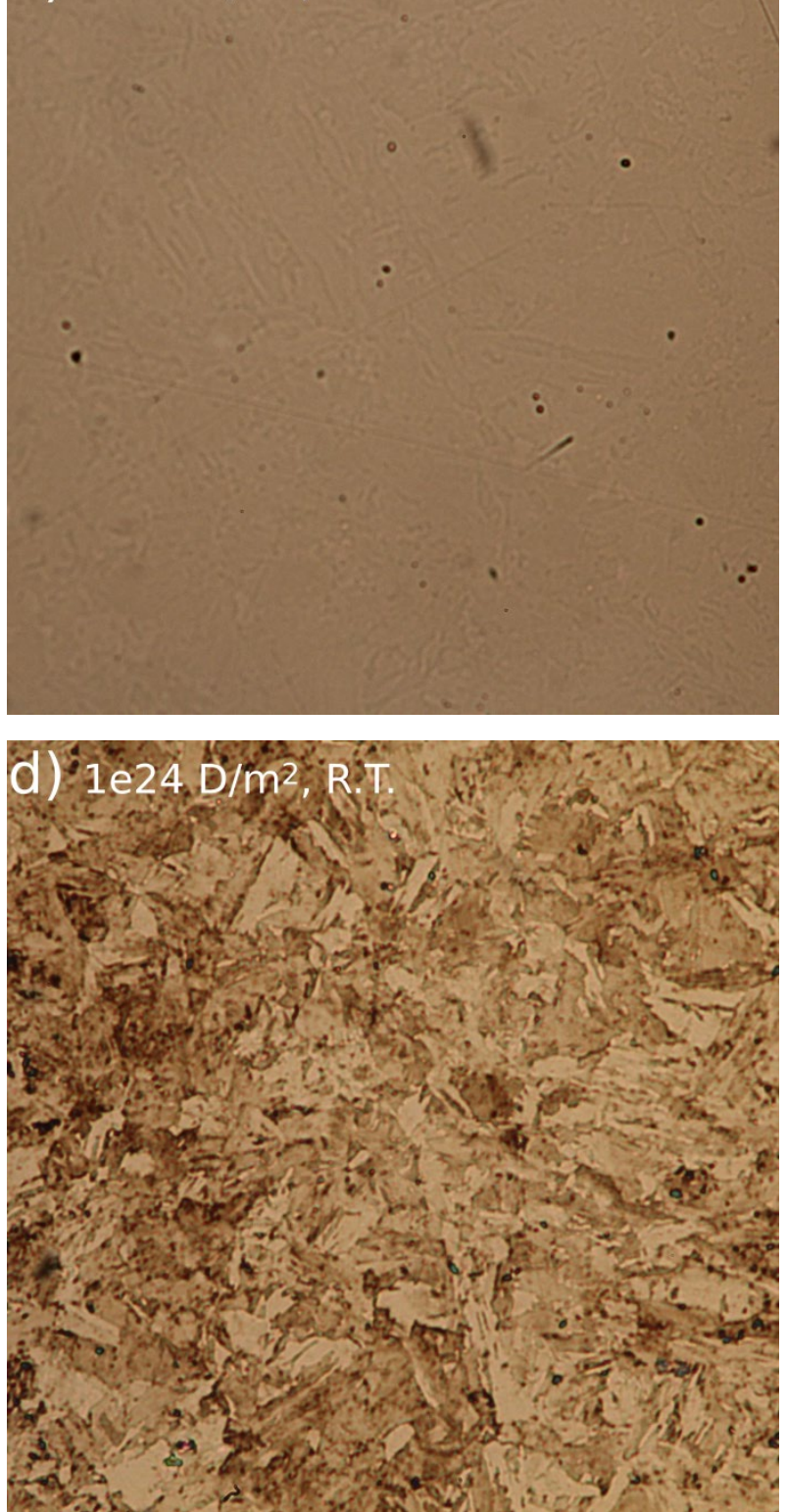

Figure 4: Photographs through an optical microscope of samples exposed to (a) no ion beam, (b) $10^{23} \mathrm{D} / \mathrm{m}^{2}$, (c) $5 \times 10^{23} \mathrm{D} / \mathrm{m}^{2}$, (d) $10^{24} \mathrm{D} / \mathrm{m}^{2}$, all at room temperature and (e) $10^{23} \mathrm{D} / \mathrm{m}^{2}$ at $900 \mathrm{~K}$. The lateral scale is the same for all images. 

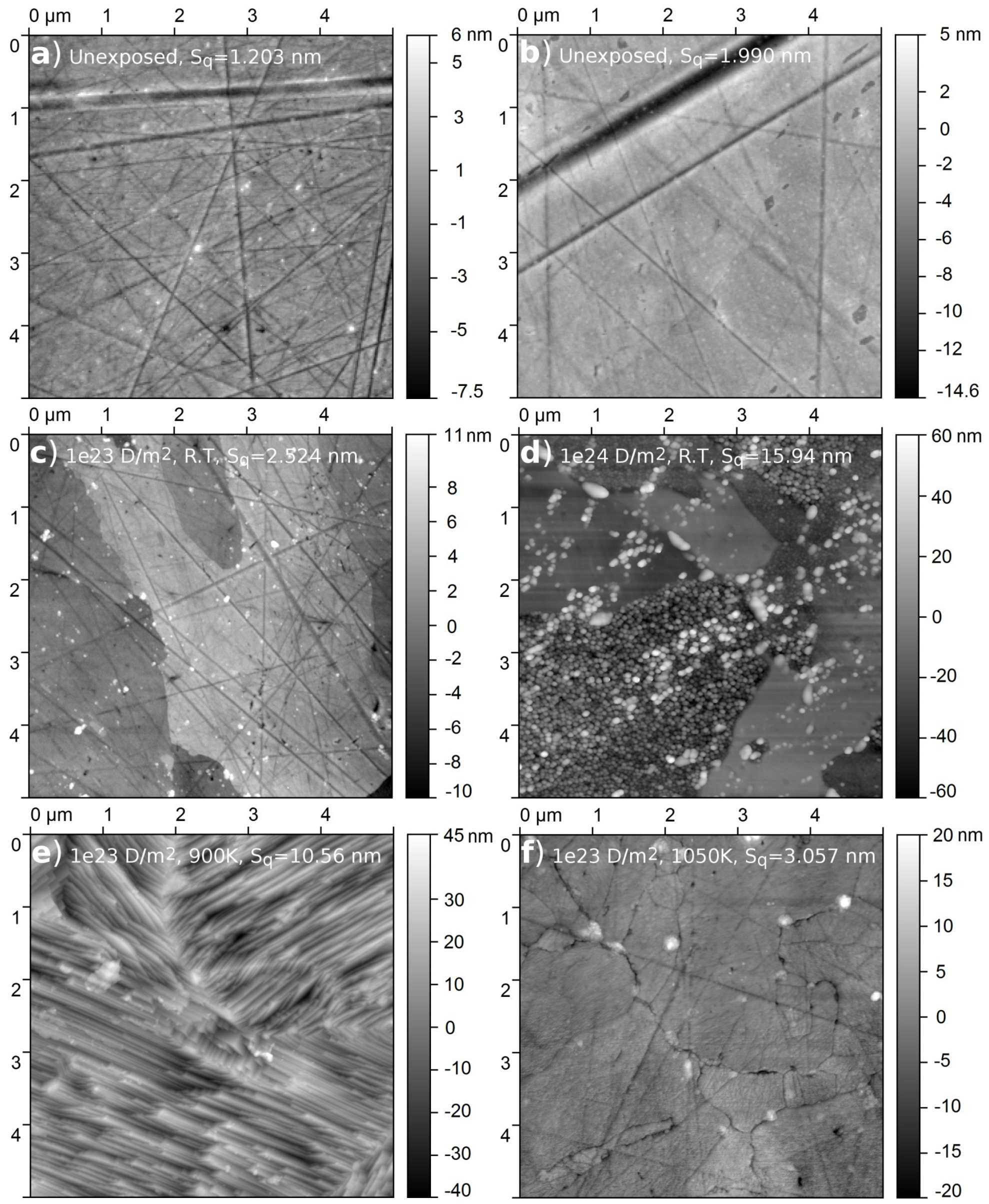
Figure 5: AFM scans of $5 \times 5 \mu \mathrm{m}^{2}$ with $256 \times 256$ pixels from unexposed samples (ab); $512 \times 512$ pixels for samples exposed to $10^{23}$ and $10^{24} \mathrm{D} / \mathrm{m}^{2}$ at room temperature (c-d) and $10^{23} \mathrm{D} / \mathrm{m}^{2}$ at $900 \mathrm{~K}$ and $1050 \mathrm{~K}$ (e-f). Deuterium particle fluence, exposure temperature and RMS surface roughness are given for each scan.

For applications of reduced activation steels like EUROFER97 in future fusion machines, exposure to oxygen will be limited. It is therefore relevant to determine whether or not surface oxidation of the samples before exposure to the SIESTA ion beam has interfered with the present sputtering experiment. We consider the simplified case of an iron-tungsten mixture, where the tungsten fraction is small, and where an iron oxide layer is present at the surface. An estimation of the sputter rate for a given element in such a mixture can be made by calculating the sputter rate that would have been obtained on a pure sample of the element in question and multiplying it by the surface fraction of the element in the mixture. This works for iron and tungsten here, assuming that their surface binding energies in the studied material do not differ significantly from those in pure samples. The sputtering yield for iron by $200 \mathrm{eV}$ deuterium ions is approximately $2 \times 10^{-2}$ at/ion [22,23], and for tungsten it is less than $10^{-4}$ at/ion since the maximum energy transfer from a $200 \mathrm{eV}$ deuteron to tungsten nucleus with which it collides is between $8.5 \mathrm{eV}$ and $8.7 \mathrm{eV}$ depending on the isotope, close to the surface binding energy of $8.6 \mathrm{eV}$ [9]. For oxygen, where the sputter yield for a pure solid sample is not a relevant quantity, we may instead take the value of between $2.5 \times 10^{-2}$ at/ion and $3 \times 10^{-2}$ at/ion for carbon from Ref. [22] and [23] as a rough approximation. The approximation is warranted as the energy transfer from a deuteron to a stationary ${ }^{12} \mathrm{C}$ nucleus in a collision is about $24 \%$ higher than that to a ${ }^{16} \mathrm{O}$ nucleus for the same recoil angle (proportional to $m_{p} m_{t} /\left(m_{p}+m_{t}\right)^{2}$, where $m_{p}$ and $m_{t}$ are the projectile and target masses), while the surface binding energies have been calculated as $7.37 \mathrm{eV}$ for carbon [22] and $5.2 \mathrm{eV}$ to $5.6 \mathrm{eV}$ for oxygen bound on a polycrystalline iron surface [24]. Assuming that the oxide layer consists of $\mathrm{Fe}_{2} \mathrm{O}_{3}$ and applying the method outlined above, we get that an incident deuterium particle fluence of $10^{23} \mathrm{D} / \mathrm{m}^{2}$ leads to removal of approximately $8 \times 10^{16}$ at $/ \mathrm{cm}^{2}$ of iron and $1.5 \times 10^{17}$ at $/ \mathrm{cm}^{2}$ of oxygen or roughly $30 \mathrm{~nm}$ of material given the depth scale conversion of $8.2 \times 10^{15}\left(\mathrm{at} / \mathrm{cm}^{2}\right) / \mathrm{nm}$ from section 3.1. The surface oxide layers on our samples, containing a few times $10^{16}$ oxygen atoms $/ \mathrm{cm}^{2}$ before ion beam exposure are thus removed within the early stages of the sample 
exposure to $10^{23} \mathrm{D} / \mathrm{m}^{2}$. For $5^{\times 10^{23}}$ and $10^{24} \mathrm{D} / \mathrm{m}^{2}$, the oxide layer is practically completely removed within a few percent of the exposure. In this discussion we have neglected the influence of tungsten, which decreases the overall sputter yield by $200 \mathrm{eV}$ deuterons impinging on an iron-tungsten alloy to less than $10^{-2}$ at/ion after exposure to $10^{24} \mathrm{D} / \mathrm{m}^{2}$ as reported in Ref. [12] and [14]. An SDTrimSP [25] simulation was performed with $200 \mathrm{eV} \mathrm{D}$ ions impinging at normal incidence on a layer with 60 at.\% O, 39.84 at.\% Fe and 0.16 at.\% W of $5 \mathrm{~nm}$ thickness, i.e. approximately $5 \times 10^{16}$ at $/ \mathrm{cm}^{2}$ in total or $3 \times 10^{16}$ at $/ \mathrm{cm}^{2}$ of oxygen, on top of a bulk with 0.4 at.\% $\mathrm{W}$ and 99.6 at.\% Fe. The simulation showed a reduction of the surface fraction of oxygen to less than $5 \%$ after exposure to $10^{22} \mathrm{D} / \mathrm{m}^{2}$, and less than $1 \%$ after approximately $2 \times 10^{22} \mathrm{D} / \mathrm{m}^{2}$, in agreement with our estimation.

Oxygen present in the chamber may potentially re-oxidize the samples during exposure to the ion beam. We evaluate the effect of re-oxidation by using an upper estimate of the partial pressure of $\mathrm{O}_{2}$ molecules: $4 \times 10^{-9} \mathrm{mbar}$. Thus, at $300 \mathrm{~K}$, the incident flux of $\mathrm{O}_{2}$ to the sample surface is $10^{16}$ molecules $/ \mathrm{m}^{2} \mathrm{~s}$ by the Hertz-Knudsen formula for an ideal gas, or $2 \times 10^{16}$ oxygen atoms $/ \mathrm{m}^{2} \mathrm{~s}$. Assuming a sticking coefficient of 1 , the oxygen fraction at the surface when adsorption is balanced by sputtering is $10 \%$, with the deuterium particle flux of $8 \times 10^{18} \mathrm{D} / \mathrm{m}^{2} \mathrm{~s}$ from section 2 and the lower estimate for the sputtering yield of $2.5 \times 10^{-2}$ at/ion from above.

When it comes to the temperature series, we note that different types of surface morphology changes have occurred for the samples exposed at 900 and $1050 \mathrm{~K}$. At $900 \mathrm{~K}$ we see a completely new surface structure, both with optical microscopy in Fig. 4 e, but more clearly with AFM in Fig. 5 e. We propose that this change is related to recrystallization occurring while the sample was exposed and partly during cooldown before removal from the chamber in which the exposure was performed. It should be noted that the morphology change is seen primarily inside the SIESTA beam footprint. The edges of the sample are more similar in appearance to an unexposed reference sample, and as such the observed surface modification is a synergistic effect of both heating and ion beam exposure. At 1050K cracking of the surface occurs. While the recrystallization corresponded to an increase in surface roughness to approximately $11 \mathrm{~nm}$ RMS, the cracking only increased it to $3 \mathrm{~nm}$. The question remains why the two samples show such different responses to ion beam 
exposure at elevated temperature. We note that the cooling down after exposure in SIESTA was not controlled, but the samples were kept in vacuum for a few minutes after which nitrogen gas was introduced. Cracking may possibly be related to thermal contraction at the sample surface depending on the cooling rate, which could help explain why cracking has occurred only on one of the samples. Despite the changes in surface structure, the tungsten and tantalum enriched layers as measured by ToFMEIS in the samples exposed at elevated temperatures are similar to what is seen at room temperature (see Fig. 1 b). The layer thickness appears to be unaffected by the increase in exposure temperature but the ToF-MEIS signal height corresponding to tungsten and tantalum is increased for the highest temperatures by a factor between 1.5 and 2 (see Fig. 1 b), indicating a similar increase of the total surface fraction of those elements.

\section{Summary and conclusions}

We have measured surface enrichment with tungsten and tantalum in samples exposed to a deuterium ion beam. The projectile energy was $200 \mathrm{eV} / \mathrm{D}$ and the flux received by the sample was between $6.7 \times 10^{18} \mathrm{D} / \mathrm{m}^{2} \mathrm{~s}$ and $9.7 \times 10^{18} \mathrm{D} / \mathrm{m}^{2} \mathrm{~s}$. Enrichment was observed at room temperature for deuterium particle fluences of $10^{23} \mathrm{D} / \mathrm{m}^{2}$ and above. The average fraction of tungsten plus tantalum to total metal content in the $2 \mathrm{~nm}$ closest to the sample surface was increased from an initial 0.0046 to $0.023,0.085$ and 0.12 respectively for fluences of $10^{23}, 5^{\times 10^{23}}$ and $10^{24}$ $\mathrm{D} / \mathrm{m}^{2}$. At $5 \times 10^{23} \mathrm{D} / \mathrm{m}^{2}$, significant surface modification accompanied the enrichment and at $10^{24} \mathrm{D} / \mathrm{m}^{2}$ the appearance of up to $40 \mathrm{~nm}$ high protrusions was observed on individual grains in the material. As such, attempts at depth profiling the enriched layer were inconclusive for the highest fluences. The ToF-MEIS spectra appeared to indicate significant enrichment down to at least $20 \mathrm{~nm}$, but it can not be excluded that this is partly a roughness effect. For $10^{23} \mathrm{D} / \mathrm{m}^{2}$, on the other hand, surface modification was limited to height variations at grain boundaries and overall surface roughness was not significantly increased by the ion beam exposure. The peak width seen in Fig. 2 is therefore not entirely a roughness effect, and we conclude that the enriched layer thickness in this case is on the order of a few $\mathrm{nm}$. Since individual grains are eroded differently on a smaller lateral scale than the size of the ToF-MEIS beam spot we interpret the result as a grain average. 
We conclude that oxide present on the samples before exposure to the deuterium ion beam has not significantly impacted the results presented here for samples exposed to fluences above $10^{23} \mathrm{D} / \mathrm{m}^{2}$. Re-oxidation during exposure due to oxygen present in the chamber may have impacted the results, however not severely, as an upper estimation of the partial pressure of oxygen in the chamber and a lower estimation of the oxygen sputtering yield, combined with a sticking coefficient of 1 yields a maximum oxygen surface fraction of $10 \%$.

Exposure to $10^{23} \mathrm{D} / \mathrm{m}^{2}$ at temperatures of $750 \mathrm{~K}$, $900 \mathrm{~K}$ and $1050 \mathrm{~K}$ gave rise to an enriched layer whose thickness was similar to that produced by exposure to the same fluence at room temperature. The fraction of tungsten and tantalum at the sample surface was, however, higher for the samples exposed at the highest temperatures compared to the one exposed at room temperature by a factor between 1.5 and 2 . The exposures at $900 \mathrm{~K}$ and $1050 \mathrm{~K}$ also produced recrystallization and cracking of the sample surface. Previous results indicate that the sputter rate of EUROFER steel by deuterium ions increases towards the value for pure iron already at 770K [9], which implies either that the enriched layer is lost due to diffusion or that an increased surface roughness counteracts the effect of surface enrichment on erosion at elevated temperatures. The statement that the enriched layer would be lost to diffusion already at $770 \mathrm{~K}$ is not supported by the present results. It should be noted, however, that the balance between diffusion and surface enrichment due to preferential sputtering is dependent on the achieved deuterium particle flux. A flux of $2 \times 10^{18} \mathrm{D} / \mathrm{m}^{2} \mathrm{~s}$, i.e. approximately a factor 4 lower than in the present study was applied in Ref. [26]. A reduced surface enrichment at 520K and loss of the enriched layer at $800 \mathrm{~K}$ is reported in that study.

\section{Acknowledgments}

We would like to thank Matthew Fielden at the AlbaNova Nanofabrication facility, Stockholm for providing substantial help with the AFM measurements. This work has been carried out within the framework of the EUROfusion Consortium and has received funding from the Euratom research and training programme 2014-2018 under grant agreement number 633053. The views and opinions expressed herein do not necessarily reflect those of the European Commission. Work was performed under work package plasma facing components (WPPFC). The operation of the 
tandem accelerator and the ion implanter at the Tandem Laboratory has been supported by infrastructure grants from the Swedish Foundation for Strategic Research (SSF) and the Swedish Research Council (VR-RFI).

\section{References}

[1] B. van der Schaaf, F. Tavassoli, C. Fazio, E. Rigal, E. Diegele, R. Lindau et al., The development of EUROFER reduced activation steel, Fusion Eng. Des. 69 (2003) 197203.

[2] L.M. Giancarli, M. Abdou, D.J. Campbell, V.A. Chuyanov, M.Y. Ahn, M. Enoeda et al., Overview of the ITER TBM Program, Fusion Eng. Des. 87 (2012) 395-402.

[3] Yu. Igitkhanov, B. Bazylev, I. Landman, L. Boccaccini, Applicability of tungsten/EUROFER blanket module for the DEMO first wall, J. Nucl. Mater. 438 (2013) S440-S444.

[4] F. Hernández, P. Pereslavtsev, Q. Kang, P. Norajitra, B. Kiss, G. Nádasi et al., A new HCPB breeding blanket for the EU DEMO: Evolution, rationale and preliminary performances, Fusion Eng. Des. 124 (2017) 882-886.

[5] R.L. Klueh, D.S. Gelles, S. Jitsukawa, A. Kimura, G.R. Odette, B. van der Schaaf et al., Ferritic/martensitic steels - overview of recent results, J. Nucl. Mater. 307-311 (2002) 455-465.

[6] G. Yu, N. Nita, N. Baluc, Thermal creep behaviour of the EUROFER 97 RAFM steel and two European ODS EUROFER 97 steels, Fusion Eng. Des. 75-79 (2005) 1037-1041.

[7] R. Schäublin, A. Ramar, N. Baluc, V. de Castro, M.A. Monge, T. Leguey et al., Microstructural development under irradiation in European ODS ferritic/martensitic steels, J. Nucl. Mater. 351 (2006) 247-260.

[8] K. Mergia, N. Boukos, Structural, thermal, electrical and magnetic properties of Eurofer 97 steel, J. Nucl. Mater. 373 (2008) 1-8. 
[9] J. Roth, K. Sugiyama, V. Alimov, T. Höschen, M. Baldwin, R. Doerner, EUROFER as a wall material: Reduced sputtering yields due to W surface enrichment, J. Nucl. Mater. 454 (2014) 1-6.

[10] V. Kh. Alimov, Y. Hatano, N. Yoshida, H. Watanabe, M. Oyaidzu, M. Tokitani et al., Surface modification and sputtering erosion of reduced activation ferritic martensitic steel F82H exposed to low-energy, high flux deuterium plasma, Nucl. Mat. Energy 7 (2016), 25-32.

[11] R. Shimizu, Preferential Sputtering, Nucl. Instr. Meth. B18 (1987), 486-495.

[12] K. Sugiyama, J. Roth, V.Kh. Alimov, K. Schmid, M. Balden, S. Elgeti et al., Erosion study of Fe-W binary mixed layer prepared as model system for RAFM steel, J. Nucl. Mater. 463 (2015) 272-275.

[13] P. Ström, P. Petersson, M. Rubel, D. Primetzhofer, S. Brezinsek, A. Kreter et al., Ion beam analysis of tungsten layers in EUROFER model systems and carbon plasma facing components. Nucl. Instr. Meth. B371 (2016), 355-359.

[14] P. Ström, D. Primetzhofer, T. Schwarz-Selinger, K. Sugiyama, Compositional and morphological analysis of FeW films modified by sputtering and heating, Nucl. Mat. Energy 12 (2017) 472-477.

[15] J.H. You, T. Höschen, G. Pintsuk, Damage and fatigue crack growth of Eurofer steel first wall mock-up under cyclic heat flux loads. Part 1: Electron beam irradiation tests, Fusion Eng. Des. 89 (2014) 284-288.

[16] M.K. Linnarsson, A. Hallén, J. Åström, D. Primetzhofer, S. Legendre, G. Possnert, New beam line for time-of-flight medium energy ion scattering with large area position sensitive detector. Rev. Sci. Instrum. 83 (2012), 095107. 
[17] P. Ström, P. Petersson, M. Rubel, G. Possnert, A combined segmented anode gas ionization chamber and time-of-flight detector for heavy ion elastic recoil detection analysis, Rev. Sci. Instrum. 87 (2016) 103303.

[18] J.P. Biersack, E. Steinbauer, P. Bauer, A particularly fast TRIM version for ion backscattering and high energy ion implantation, Nucl. Instr. Meth. B61 (1991) 7782.

[19] K. Arstila, J. Julin, M.I. Laitinen, J. Aalto, T. Konu, S. Kärkkäinen et. al., Potku New analysis software for heavy ion elastic recoil detection analysis, Nucl. Instrum. Meth. B331 (2014) 34-41.

[20] M. Rasiński, S. Möller, J. Steffens, B. Unterberg, K. Sugiyama, T. SchwarzSelinger et al., Morphology and composition of Fe-W coatings after deuterium plasma exposure as a model system for RAFM steels, Phys. Scr. T167 (2016) 014013.

[21] M. Rasiński, A. Kreter, S. Möller, T. Schlummer, Y. Martynova, S. Brezinsek et al., The microstructure of reduced activation ferritic/martensitic (RAFM) steels exposed to D plasma with different seeding impurities, Phys. Scr. T170 (2017) 014036.

[22] N. Matsunami, Y. Yamamura, Y. Itikawa, N. Itoh, Y. Kazumata, S. Miyagawa et al., Energy dependence of the ion-induced sputtering yields of monoatomic solids, At. Data Nucl. Data Tables 31 (1984) 1-80.

[23] W. Eckstein, Sputtering yields, in: R. Behrisch, W. Eckstein (Eds.), Topics in Applied Physics 110, Sputtering by Particle Bombardment, Experiments and Computer Calculations from Threshold to $\mathrm{MeV}$ Energies, Springer-Verlag $\mathrm{GmbH}$, Heidelberg, Germany, 2007, pp. 33-186, doi: 10.1007/978-3-540-44502-9.

[24] K.W. Frese Jr., Calculation of surface binding energy for hydrogen, oxygen and carbon atoms on metallic surfaces, Surf. Sci. 182 (1987), 85-97. 
[25] A. Mutzke, R. Schneider, W. Eckstein, R. Dohmen, SDTrimSP Version 5.0o, Garching: Max-Planck-Institut für Plasmaphysik, report (2011).

Available online: http://hdl.handle.net/11858/oo-001M-oooo-o026-EAF9-A, accessed 2018-05-02.

[26] H.R. Koslowski, S.R. Bhattacharyya, P. Hansen, Ch. Linsmeier, M. Rasiński, P. Ström, Temperature-Dependent In-Situ LEIS Measurement of W Surface Enrichment by 250 eV D Sputtering of Eurofer, Preprint, arXiv:1803.0180o. 